\title{
OPTICAL SENSING OF MAGNETIC FIELDS
}

M. A. Butler and S. J. Martin

Microsensor Division 1142

Sandia National Laboratories

Albuquerque, New Mexico 87185

\section{Introduction}

The development of optical fiber technology has spurred the development of a wide range of optical sensors for many physical parameters.[1] Interferometric sensors where the fiber is coated with a magnetic material are now capable of sensitivities in the microguass range [2] with potential for further improvement. These sensors, however, have bandwidths limited to the kilohertz range because of the nature of the magnetostrictive coating materials. The recent development of new magnetic semiconductors which show very large Faraday rotations make possible moderately sensitive devices (milliguass) with very large bandwidths (gigahertz).

In this talk, we would like to explore the limitations on optical sensing of magnetic fields using the Faraday effect imposed by the properties of the sensing material. Emphasis will be given to factors limiting the bandwidth of these devices.

\section{Faraday Effect}

The Faraday effect involves modification of the dielectric response of a material by an applied magnetic field. This can come about through the modification of the electronic transition energies, the matrix elements and/or the populations of the spin split ground state. The first two factors normally lead to a "diamagnetic" or temperature independent Faraday effect and the last factor leads to a "paramagnetic" or temperature dependent Faraday effect. Simple models of these effects [3] show that the Faraday effect can be maximized by using light with a photon energy close to the electronic transition energy and by having a material with a strongly paramagnetic groundstate. This is the reason for the use of rare earth doped glasses.

The dilute magnetic semiconductors are a relatively new class of materials which have been found to show very large Faraday effects. [4] These materials are exemplified by the $\mathrm{Cd}(1-\mathrm{x}) \mathrm{Mn}(\mathrm{x}) \mathrm{Te}$ system which allows values of $x$ up to 0.7 without a phase transition. In this material the bandgap of the semiconductor varies linearly with $x$ allowing the tuning of the electronic transition energy to match the light source used. The Faraday effect is approximately proportional to the Mn concentration.

\section{Experimental Results}

Faraday rotation measurements have been made on several alloy compositions at a number of different wavelengths and at frequencies up to 10 gigahertz. These measurements were made by incorporating the sensing material into a microstripline as shown in Figure 1. R.F. power of

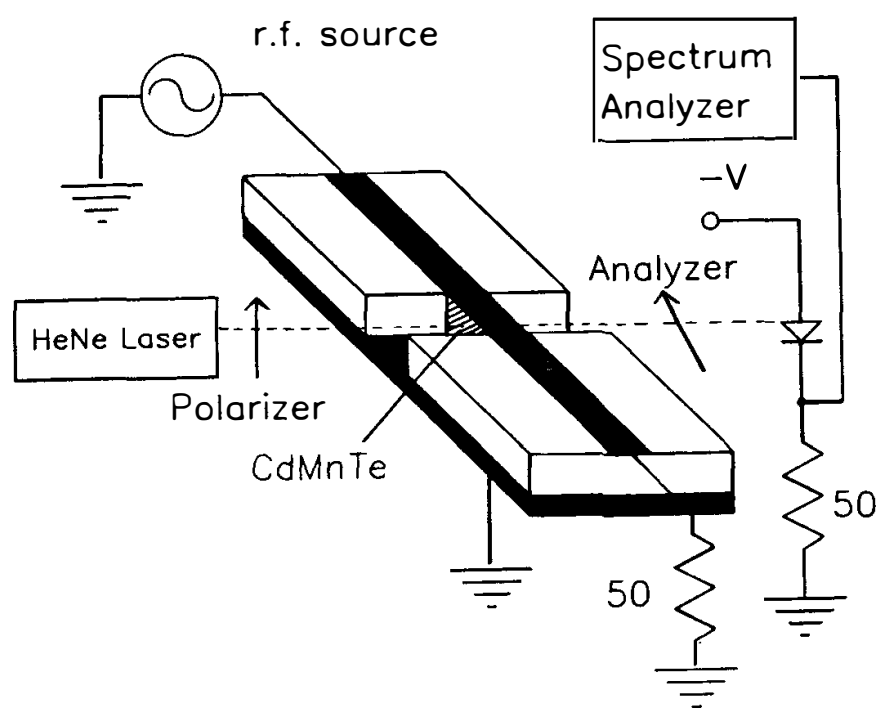

one watt passing along the microstrip produces an rms magnetic field in the sample of about 100 milligauss. By passing a linearly polarized light beam through the sample at right angles to the microstrip the polarization direction of the beam is modulated at the R.F. frequency. This modulation is detected by a polarizing analyzer, which converts the polarization modulation to amplitude modulation, and a fast photodiode. The frequency response of the $\mathrm{Cd}(.9) \mathrm{Mn}(.1) \mathrm{Te}$ sample is shown in Figure 2. Other compositions show a

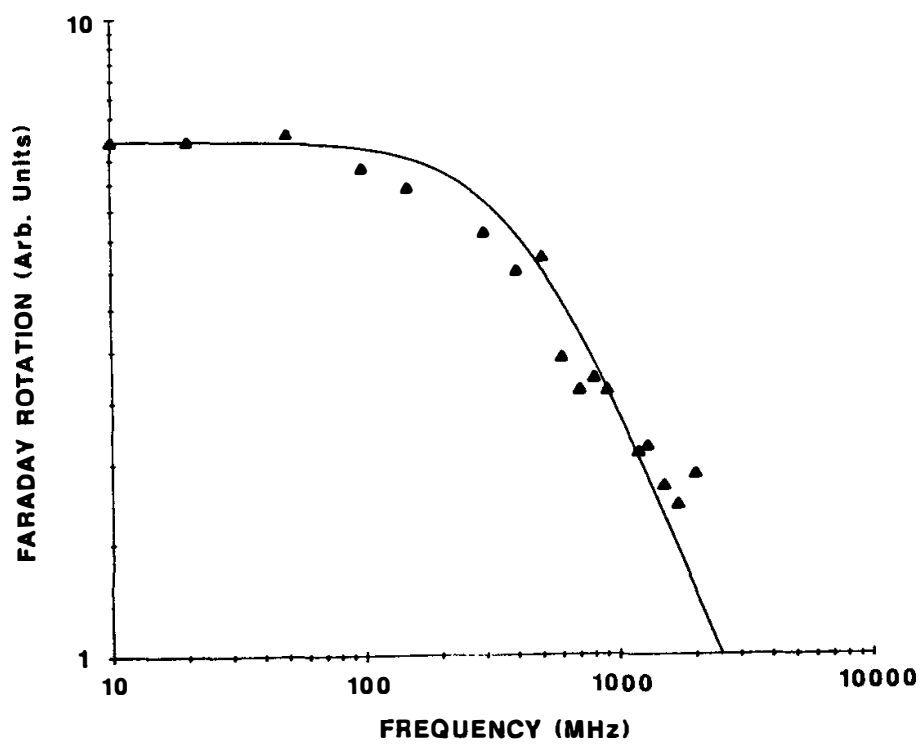

similar functional dependence to the frequency response with the rolloff frequency increasing with Mn concentration. 
The time (and thus the frequency) dependence of the Faraday effect can easily be shown to reflect the time dependence of the ground state magnetization. Thus, the frequency response of Faraday effect magnetic field sensors will be determined by the spin dynamics. It is well known [5] that for small applied fields the frequency response of a collection of spins is well described by the modified Bloch equations. The solution yields the functional form for the frequency response shown by the solid line in Figure 2.

$$
M(\omega)=\frac{M_{0}}{\sqrt{1+(\omega T)^{2}}}
$$

The time constant $\mathrm{T}$ reflects the physical processes which allow the magnetization to respond to the applied magnetic field. In the magnetically dilute limit, the ground state magnetization can only grow by spin flips which require that energy be given up to the lattice. Thus the response is determined by "spin-lattice" relaxation processes. In the magnetically dense limit the magnetization is not a conserved quantity at low fields and the magnetization can be changed without transfering energy out of the spin system. In this case the response is determined by "spin-spin" relaxation processes. This is the appropriate description for the CdMnTe system concentrations considered.

\section{Conclusions}

The dilute magnetic semiconductors offer great possibilities as sensor materials for Faraday effect magnetic field sensors. The effect is very large and can be optimized for the light source to be used because of the tuneability of the bandgap. The frequency response is determined by the spin dynamics of the Mn spins and will allow bandwidths of several gigahertz.

\section{$\underline{\text { References }}$}

1. T. G. Giallorenzi, J. A. Bucaro, A. Dandridge, G. H. Sigel, J. H. Cole, S. C. Rashleigh and R. Priest, "Optical Fiber Sensor Technology," J. Quantum Electronics, Vol. QE-18, 626, 1982.

2. C. J. Nielsen, "All Fiber Magnetometer with Magnetic Feedback Compensation," Proc. of SPIE, Vol. 566, 286, 1985.

3. C. J. Ballhausen, Introduction to Ligand Field Theory, New York: McGraw Hill, 1962, p. 211.

4. J. K. Furdyna, "Dilute Magnetic Semiconductors: An Interface of Semiconductor Physics and Magnetism," J.Appl. Phys., Vol. 53, 7637, 1982.

5. A. Abragam, The Principles of Nuclear Magnetism, London: Oxford University Press, 1961 p. 53. 